\title{
Continuous Sampling Plan in Preventing Defects with Risk Evaluation in Production and Sales System
}

\author{
Sundar Viswanathan \\ Senior Testing Engineer, ANSYS Inc., \\ 2600,ANSYS Drive, Canonsburg, PA 15317
}

\begin{abstract}
This paper studies a continuous sampling plan in preventing entries of defective products produced for sales using queueing theory methods. The sampling plan considered here has three inspection modes. All the products are inspected in mode I and in modes II and III the products are inspected with some probability $\mathrm{c} \geq 0$ and $\mathrm{d}>0$ respectively in the modes in order to avoid high cost of inspecting all products produced. Matrix methods are used for studying the stock level probabilities and various performance and risk measures including the rate of entry of defectives, expected defective products in the stock, standard deviation and the coefficient of variation are presented. Stationary stock level probabilities are derived using iterated rate matrix. Two special cases with $\mathrm{c}>0$ and $\mathrm{d}=1$ and with $\mathrm{c}=0$ and $\mathrm{d}>0$ are considered. Numerical cases are treated to note the significance of the continuous sampling plan in reducing the entry rates. The expected defective products in the stock for sales and all the risk measures are listed and discussed.
\end{abstract}

\section{General Terms}

Production, Sales, Defective Product, Continuous Sampling Plan, Queueing Theory

\section{Keywords}

Stationary Probability, Block Partitioned Methods, Infinitesimal Generator, Expected Defective Products, Defective Entry Rates.

\section{INTRODUCTION}

It has become very common that when products are manufactured by a machine some products are found to be good and some are defective. Because of the cost involved in inspecting all the products may be very high, various inspection policies are adopted to reduce this cost. Continuous Sampling Plans (CSP) are introduced to reduce the inspection cost to a manageable level. This may filter the entry of defective products to some extent but not in full. It is of interest to policy makers to know in the long run, the expected number of products and defective products in the stock when a CSP is adopted and the risk values for the same. The CSP to be followed must have relation with number of good products or defectives noticed. The CSP studied in this paper has three inspection modes and the modes change depending on good and defective items found during inspection. It is a combination of the CSP mentioned in Dodge [1], G.K Mytalas and M. A Zazanis [2] and A.H.Bowker [3] where CSP was considered to have two inspection modes only with non-zero probability in mode II which is a restriction. The risk involved in the CSP are presented and measured by finding the rate of entry of defectives, the expected defective products in the stock, the standard deviation and the coefficient of variation. The products produced form a queue for sales. For studies on queues one may refer to Ivo Adan and Jacques Resing [4]. Ken block [5] and James c. Cox and Vjollca Sadiraj [6] have discussed the coefficient of variation in detail. There are many measures of risk, one may refer Rockefeller [7]. This paper examines the performance of the CSP in the case of a continuous time Markov chain model and presents results identifying Neuts [8] matrix structures. For queueing theory numerical results one may refer to Bini, Latouche and Mein [9]. Matrix analytic methods have been treated by Latouche and Ramaswami [10].

This paper considers production by a machine which produces good and defective products. The products are inspected for sales. The CSP considered here has three inspection modes. In inspection mode I, every single product is inspected until k consecutive good products are found. At this point the CSP changes its inspection to mode II of inspecting the next $r$ products where every product is inspected with probability $\mathrm{c} \geq$ 0 until a defective product appears. When a defective one appears, the CSP changes its inspection mode to I and if no defective product is noticed in those $r$ products, the CSP changes its inspection mode to III where the product is inspected with probability $\mathrm{d}>0$. If the product is rejected, the CSP changes its inspection mode to I and if it is not rejected then the CSP changes its inspection mode to II. In the above CSP, the probabilistic inspection procedures reduce the number of inspections considerably with a view to reduce the inspection cost.

The paper is organized in the following manner. In Sections (2) the stock level probabilities are derived using matrix geometric approach when the CSP is adopted. In Section 3, the performance measures and various stock level probabilities are presented in the stationary case. The expected stock level, the expected defective stocks, the standard deviation, the rate of entry of defectives, the variance and the co-efficient of variation as measures are obtained. In section (4), two models are presented. In Model (A), the probabilities considered in inspection modes II and III are $\mathrm{c}>$ 0 and $\mathrm{d}=1$ and Model (B) studies the case $\mathrm{c}=0$ and $\mathrm{d}>0$. In section (5) numerical cases are treated.

\section{STEADY STATE ANALYSIS UNDER CONTINUOUS SAMPLING PLAN WITH THREE MODES}

\subsection{Assumptions}

(i) The production time of a product has exponential distribution with parameter $\lambda$ and each product produced is good with probability $\mathrm{p}$ and is defective with probability $\mathrm{q}$ where $\mathrm{p}+\mathrm{q}=1$.

(ii) The CSP has three inspection modes. In mode I every single product is inspected until $\mathrm{k}$ consecutive good products are found. At this point, the CSP changes its inspection mode to II of inspecting the next $r$ products where every product is inspected with probability $c \geq 0$ until a defective product appears. When a defective one appears, the CSP changes to inspection mode I and if no defective product is noticed in those $r$ products, the CSP changes its inspection mode to III 
where the product is inspected with probability $d>0$. If it is rejected, the inspection mode $\mathrm{I}$ is started and if it is not rejected then inspection mode II begins. The products found to be defective in the inspection modes I, II and III are rejected and the products which are not rejected are ready for sales.

(iii)The products are sold at sale epochs with the inter occurrence time between two consecutive sale epochs has exponential distribution with parameter $\mu$. At any sale epoch with probability $\alpha$, a normal sale occurs and with probability $\beta$, a catastrophic sale occurs, where $\alpha+\beta=1$. In a normal sale $\mathrm{N}$ products are sold at a time with probability $\mathrm{P}(\mathrm{N}=\mathrm{i})=p_{i}$, for $1 \leq \mathrm{i} \leq \mathrm{m}$ where $\sum_{1}^{m} p_{i}=1$. When $\mathrm{n}$ products $\mathrm{n}<\mathrm{m}$ are available, then i products are sold with $\mathrm{P}(\mathrm{N}=\mathrm{i})=p_{i}$, for $1 \leq \mathrm{i}$ $\leq \mathrm{n}-1$ and $\mathrm{n}$ products are sold with probability $\sum_{n}^{m} p_{i}$, as sales are only for available number $\mathrm{n}$ of products. When the catastrophic sale occurs, all the products are sold.

\subsection{Analysis}

For studying the above model, the state of the system of the continuous time Markov chain $X(t)$ may be defined as follows.

$X(t)=\{(n, i, j): 0 \leq n<\infty$; for $i=1,1 \leq j \leq k$; for $i=2,1 \leq j \leq$ r $\} \quad U\{(n, 3): 0 \leq n<\infty\}$.

(1)

The system is in the state $(n, 1, j)$ when $n$ products are available for sales for $0 \leq \mathrm{n}<\infty$, and the CSP inspection mode is $I$ and $(j-1)$ consecutive products are not rejected in inspection mode $\mathrm{I}$, for $1 \leq \mathrm{j} \leq \mathrm{k}$. The system is in the state (n, 2 ,j) when $n$ products are available for sales, for $0 \leq n<\infty$ and the CSP inspection mode is II and ( $\mathrm{j}-1)$ consecutive products are not rejected in inspection mode II, for $1 \leq \mathrm{j} \leq \mathrm{r}$. The system is in the state $(n, 3)$ when $n$ products are available for sales and the CSP inspection mode is III for $0 \leq \mathrm{n}<\infty$.

Let the probability generating function of $\mathrm{N}$, the number of products sold in a normal sale be $\varphi(\mathrm{r})=\sum_{i=1}^{m} p_{i} r^{i}$.

(2)

Let the generating function of the survivor probability $\mathrm{P}(\mathrm{N}>$ i) $=P_{i}=1-\sum_{j=1}^{i} p_{j}$

(3) be given by $\varnothing(\mathrm{r})=\sum_{i=1}^{m-1} P_{i} r^{i}$

(4)

The relation between them is $\emptyset(r)=(r / 1-r) \varphi(r)$.

(5)

continuous time Markov chain describing the model has infinitesimal generator $\mathrm{Q}$ of infinite order which can be presented in block partitioned form with each block is of order $(\mathrm{k}+\mathrm{r}+1)$. The infinitesimal generator of the model is given below. $\mathrm{Q}=$

$\left[\begin{array}{ccccccccc}B_{1} & A_{0} & 0 & 0 & \cdots & . & . & . & \cdots \\ B_{2} & A_{1} & A_{0} & 0 & \cdots & . & . & . . & \cdots \\ B_{3} & A_{2} & A_{1} & A_{0} & \cdots & . & . & . & \cdots \\ \vdots & \vdots & \vdots & \vdots & \vdots & \vdots & \vdots & \vdots & \vdots: \vdots \\ B_{m} & A_{m-1} & A_{m-2} & A_{m-3} & \cdots & 0 & 0 & . & \cdots \\ B_{m+1} & A_{m} & A_{m-1} & A_{m-2} & \cdots & 0 & 0 & . & \cdots \\ B_{m+2} & A_{m+1} & A_{m} & A_{m-1} & \cdots & A_{0} & 0 & 0 & \cdots \\ B_{m+2} & 0 & A_{m+1} & A_{m} & \cdots & A_{1} & A_{0} & 0 & \cdots \\ B_{m+2} & 0 & 0 & A_{m+1} & \cdots & A_{2} & A_{1} & A_{0} & \cdots \\ \vdots & \vdots & \vdots & \ddots & \ddots & \ddots & \ddots & \ddots & \ddots\end{array}\right](6)$

Its component- block matrices are listed below. Let $A_{0}$ be a matrix of order $\mathrm{k}+\mathrm{r}+1$ with only one non-zero element in each row as given below.

$$
\begin{aligned}
A_{0} & =\left[\begin{array}{cccccccc}
0 & \lambda p & 0 & \cdots & 0 & 0 & \cdots & 0 \\
0 & 0 & \lambda p & \cdots & 0 & 0 & \cdots & 0 \\
0 & 0 & 0 & \cdots & 0 & 0 & \cdots & 0 \\
\vdots & \vdots & \vdots & \ddots & \vdots & \vdots & \vdots & \vdots \\
0 & 0 & 0 & \cdots & \lambda p & 0 & \cdots & 0 \\
0 & 0 & 0 & \cdots & 0 & \lambda \gamma & \cdots & 0 \\
\vdots & \vdots & \vdots & \vdots & \vdots & \vdots & \ddots & \vdots \\
0 & 0 & 0 & \cdots & 0 & 0 & \cdots & \lambda \gamma \\
0 & 0 & 0 & \cdots & \lambda \delta & 0 & \cdots & 0
\end{array}\right] \\
B_{1} & =\left[\begin{array}{cccccccc}
-\lambda p & 0 & \cdots & 0 & 0 & \cdots & 0 & 0 \\
\lambda q & -\lambda & \cdots & 0 & 0 & \cdots & 0 & 0 \\
\vdots & \vdots & \vdots & \vdots & \vdots & \vdots & \vdots & \vdots \\
\lambda q & 0 & \cdots & -\lambda & 0 & \cdots & 0 & 0 \\
\lambda q c & 0 & \cdots & 0 & -\lambda & \cdots & 0 & 0 \\
\vdots & \vdots & \cdots & \vdots & \vdots & \ddots & \vdots & \vdots \\
\lambda q c & 0 & \cdots & 0 & 0 & \cdots & -\lambda & 0 \\
\lambda q d & 0 & \cdots & 0 & 0 & \cdots & 0 & -\lambda
\end{array}\right]
\end{aligned}
$$

In (7), $\gamma=1$ - q c, $\left(A_{0}\right)_{k+r+1, k+1}=\lambda \delta$ and $\delta=1$ - $\mathrm{q}$ d. The first super diagonal elements of $A_{0}$ are positive. All its others elements are $=0$.The matrix $B_{1}$ has negative diagonal elements and except the first column all its off diagonal elements are equal to zero. Its elements are placed as follows. $\left(B_{1}\right)_{i, 1}=\lambda \mathrm{q}$ for $2 \leq \mathrm{i} \leq \mathrm{k}$ and $\left(B_{1}\right)_{k+r+1,1}=\lambda \mathrm{qd} ;\left(B_{1}\right)_{i, 1}=\lambda \mathrm{q} \mathrm{c}$, for $\mathrm{k}+1 \leq$ $\mathrm{i} \leq \mathrm{k}+\mathrm{r}$. Let $\mathbf{I}$ be identity matrix of order $(\mathrm{k}+\mathrm{r}+1)$. Then let $A_{1}=B_{1}-\mu \mathbf{I}$ and $A_{j+1}=\alpha \mu p_{j} \mathbf{I}$ for $1 \leq \mathrm{j} \leq \mathrm{m}$;

(9) $B_{2}=\mu \mathbf{I} ; B_{j}=\mu\left(P_{j-2} \alpha+\beta\right) \mathbf{I}$ for $3 \leq \mathrm{j} \leq \mathrm{m}+1 ; B_{m+2}=\mu \beta \mathbf{I}$.

generator is $\mathrm{Q}^{\prime}=\sum_{i=0}^{m+1} A_{i}+B_{m+2}$

The basic system

This is also an infinitesimal generator of a continuous time Markov chain with finite state space and the matrix is of order $\mathrm{k}+\mathrm{r}+1$. The finite generator matrix $\mathrm{Q}^{\prime}$ is given by $\mathrm{Q}^{\prime}=$

$\left[\begin{array}{cccccccccc}-\lambda p & \lambda p & 0 & \cdots & 0 & 0 & 0 & \cdots & 0 & 0 \\ \lambda q & -\lambda & \lambda p & \cdots & 0 & 0 & 0 & \cdots & 0 & 0 \\ \lambda q & 0 & -\lambda & \cdots & 0 & 0 & 0 & \cdots & 0 & 0 \\ \vdots & \vdots & \vdots & \vdots & \vdots & \vdots & \vdots & \vdots & \vdots & \vdots \\ \lambda q & 0 & 0 & \cdots & -\lambda & \lambda p & 0 & \cdots & 0 & 0 \\ \lambda q c & 0 & 0 & \cdots & 0 & -\lambda & \lambda \gamma & \cdots & 0 & 0 \\ \vdots & \vdots & \vdots & \vdots & \vdots & \vdots & \vdots & \vdots & \vdots & \vdots \\ \lambda q c & 0 & 0 & \cdots & 0 & 0 & 0 & \cdots & -\lambda & \lambda \gamma \\ \lambda q d & 0 & 0 & \cdots & 0 & \lambda \delta & 0 & \cdots & 0 & -\lambda\end{array}\right]$

(12)

In the above matrix $\left(Q^{\prime}\right)_{k+r+1, k+1}=\lambda \delta ;\left(Q^{\prime}\right)_{i, 1}=\lambda \mathrm{q}$, for $2 \leq$ $\mathrm{i} \leq \mathrm{k} ;\left(Q^{\prime}\right)_{i, 1}=\lambda \mathrm{q} \mathrm{c}$, for $\mathrm{k}+1 \leq \mathrm{i} \leq \mathrm{k}+\mathrm{r}$, and $\left(Q^{\prime}\right)_{k+r+1,1}=$ $\lambda q d$. The production and inspection modes for products are governed by the matrix Q'. The transition rates of the finite continuous time Markov chain governed by inspection mode $\mathrm{I}$ are given in rows $\mathrm{i}$ for $1 \leq \mathrm{i} \leq \mathrm{k}$; the rows $\mathrm{i}$ for $\mathrm{k}+1 \leq \mathrm{i}$ $\leq \mathrm{k}+\mathrm{r}$ present the transition rates when inspection mode is II. The last row corresponds to the inspection mode III. Let $w$ be the steady state probability vector of Q'. Then $\mathrm{w} \mathrm{Q}^{\prime}=0$ and $\mathrm{w}$ $\mathrm{e}=1$.

The components of vector $\mathrm{w}=\left(w_{1}, w_{2}, w_{3}, \ldots w_{k+r+1}\right)(14)$ are

$$
\begin{array}{lrr}
w_{k+r+1}=\left[\gamma^{r} p^{k}(1-\gamma) \mathrm{q}\right] /\left[(1-\gamma)\left(1-p^{k}\right)\right. & \left(1-\delta \gamma^{r}\right)+\mathrm{q} p^{k}\left(1-\gamma^{r+1}\right) \\
\mathrm{l} \quad w_{i}=(1 / \mathrm{p})^{k-i-1}\left[\left(\frac{1}{\gamma^{r}}\right)-\delta\right] w_{k+r+1}, & \text { for } 1 \leq \\
\mathrm{i} \quad \mathrm{k} & \text { for } \mathrm{k}+1 \leq \mathrm{i}<\mathrm{k}+\mathrm{r}
\end{array}
$$$$
\text { (15) }
$$

The fraction of products that are inspected is then given by

$\mathrm{f}=1-\quad \sum_{i=1}^{r+1} w_{k+i}+\mathrm{c} \quad \sum_{i=1}^{r} w_{k+i}+\mathrm{d} \quad w_{k+r+1} \quad$. $\quad$ (16) Whereas the defective products that go undetected as a fraction of all parts is, (1-f) $\mathrm{q}=\mathrm{q}(1-\mathrm{c}) \sum_{i=1}^{r} w_{k+i}+\mathrm{q}(1-$ 
d) $w_{k+r+1}$. (17) The stability condition for the existence of a stationary distribution for the Markov chain given by $\mathrm{Q}$ by Neuts [8] is $\mathrm{w} A_{0} \mathrm{e}<\mathrm{w}\left[\sum_{j=2}^{m+1}(j-1) A_{j}\right] \mathrm{e}$. This gives from (7) and (9) $\quad \lambda\left[\mathrm{p}+\mathrm{q}(1-\mathrm{c}) \sum_{i=1}^{r} w_{k+i}+\mathrm{q}(1-\right.$ d) $\left.w_{k+r+1}\right]<\alpha \mu \quad \mathrm{E} \quad(\mathrm{N}) \quad$ (18). Here $\mathrm{E}(\mathrm{N})$ is the expected number of sales when the sale is normal. Let $\pi(n, 1, j)$, for $1 \leq \mathrm{j} \leq \mathrm{k} ; \pi(\mathrm{n}, 2, \mathrm{j})$, for $1 \leq \mathrm{j} \leq \mathrm{r}$ and $\pi(\mathrm{n}, 3)$ for $0 \leq \mathrm{n}<\infty$ be the stationary probability of the states listed in (1) and let $\pi_{n}$ be the vector of type $1 \times(\mathrm{k}+\mathrm{r}+1)$ $\pi_{n}=(\pi(\mathrm{n}, 1,1), \quad \pi(\mathrm{n}, 1,2), \quad \pi(\mathrm{n}, 1,3), \ldots ., \pi(\mathrm{n}, 1, \mathrm{k}), \quad \pi(\mathrm{n}, 2,1)$, $\pi(\mathrm{n}, 2,2), \ldots . ., \quad \pi(\mathrm{n}, 2, \mathrm{r}), \quad \pi(\mathrm{n}, 3) \quad$. (19)

The stationary probability vector $\pi=\left(\pi_{0}, \pi_{1}, \pi_{3}, \ldots \ldots \ldots \ldots . . ..\right)$ satisfies the equations $\pi \mathrm{Q}=0$, and $\pi \mathrm{e}=1$. (20)

From (20) the following are obtained $\sum_{0}^{m} \pi_{i} B_{i+1} \quad+\quad \sum_{i=1}^{\infty} \pi_{m+j} B_{m+2} \quad=\quad 0$.

(21)

$\pi_{n-1} A_{0}+\pi_{n} A_{1}+\pi_{n+1} A_{2}+\ldots \ldots \ldots . .+\pi_{n+m} A_{m+1}=0, \mathrm{n} \geq 1$. (22)

Introducing the rate matrix $\mathrm{R}$ as the minimal non-negative solution of the non-linear matrix equation $A_{0}+\mathrm{R} A_{1}+R^{2} A_{2}+R^{3} A_{3}+\ldots \ldots \ldots+R^{m+1} A_{m+1}=0$, (23)

it can be proved that (Neuts [8]) $\pi_{n}$ satisfies for $\mathrm{n} \geq 1$, $\pi_{n}=\pi_{0} R^{n} \quad$ for $\mathrm{n} \geq 1$. (24)

The vector $\pi_{0}$ satisfies the following using (20), (21) and (24).

$\pi_{0}\left(B_{1}+\sum_{J=1}^{m} R^{j} B_{j+1}+\sum_{J=1}^{\infty} R^{m+j} B_{m+2}\right)=0$ (25)

$\begin{array}{lllllll}\pi_{0} & (\mathbf{I} & - & \mathrm{R} & )^{-1} & \mathrm{e} & =1 .\end{array}$ (26)

Equation (25) may be used to find $\pi_{0}$ subject to a multiplicative constant and the constant may be evaluated using equation (26). The matrix $\mathrm{R}$ is computed by substitutions in the iteration $\mathrm{R}(0)=0 ; \mathrm{R}(\mathrm{n}+1)=-A_{0} A_{1}^{-1}-\sum_{j=2}^{m+1} R^{j}(\mathrm{n}) A_{j} A_{1}^{-1}, \quad \mathrm{n} \geq 0$. (27)

The iteration may be terminated to get a solution of $\mathrm{R}$ when $\|\mathrm{R}(\mathrm{n}+1)-\mathrm{R}(\mathrm{n})\|<\varepsilon$ where $\varepsilon$ is a small number.

\section{PERFORMANCE MEASURES}

(i) The probability of the stock level is $n$ with various inspection modes and stages can be seen from $\pi_{n}=(\pi(\mathrm{n}, 1,1), \quad \pi(\mathrm{n}, 1,2), \quad \pi(\mathrm{n}, 1,3), \ldots ., \pi(\mathrm{n}, 1, \mathrm{k}), \quad \pi(\mathrm{n}, 2,1)$, $\pi(\mathrm{n}, 2,2), \ldots . \pi(\mathrm{n}, 2, \mathrm{r}), \pi(\mathrm{n}, 3))$ and $\pi_{n}=\pi_{0} R^{n}$ as given by (24).

(ii)P (Stock level is $\mathrm{n})=\pi_{n}$ e for $\mathrm{n} \geq 0$ and the probability of empty stock is $\pi_{0} \mathrm{e}$ as derived in (25) and (26) using (27). (iii)The expected stock level in the steady state may be seen as

$\mathrm{E}(\mathrm{S})=\sum_{0}^{\infty} n \pi_{n} e=\pi_{0} \quad(\mathbf{I} \quad-\mathrm{R} \quad)^{-2} R \quad$ e. (28)

(iv)The expected number of defective stocks in the steady state level is given by using (16) as $\mathrm{E} \quad$ (Defective stocks) $=(1-\mathrm{f}) \quad \mathrm{q} \quad \mathrm{E}(\mathrm{S})$. (v)The second moment of queue length is $\mathrm{E}\left(S^{2}\right)=\pi_{0}(\mathbf{I}-\mathrm{R})^{-3} 2 R^{-2} \mathrm{e}+\pi_{0}(\mathbf{I}-\mathrm{R})^{-2} \mathrm{Re}$ (30) and the variance is given by $\operatorname{VAR}(\mathrm{S})=$ $\pi_{0}(\mathbf{I}-\mathrm{R})^{-3} 2 R^{-2} \mathrm{e}+\pi_{0}(\mathbf{I}-\mathrm{R})^{-2} \operatorname{Re}-\left(\pi_{0}(\mathbf{I}-\mathrm{R})^{-2} R \mathrm{e}\right)^{2}$ (31) (vi)The co-efficient of variation $\mathrm{CV}=\frac{\sigma_{S}}{E(S)} \quad$ may be written using (28) and (31) taking square root for variance.

(vii) The rate of entry of defectives when the CSP is adopted using (17) is, $\lambda(1-\mathrm{f}) \mathrm{q}=\lambda \mathrm{q}(1-\mathrm{c}) \sum_{i=1}^{r} w_{k+i}+\lambda \mathrm{q}(1-\mathrm{d}) w_{k+r+1}$ where $\lambda$ is the arrival rate and the rate of production of defective products is $\lambda$ q. (viii)The risk measures for the CSP under consideration are, namely, the rate of entry of defectives, the expected defective stocks, the standard deviation, and the coefficient of variation based on which the effects of the probabilities considered at the CSP modes II and III may be judged. (ix)The calculation of steady state probabilities of stock level is required to know the fraction of catastrophic sales rate or normal sales rate or the rate of entry of defective product at various stock levels

\section{SPECIAL CASES}

\subsection{Model (A) Probabilities $\mathrm{c}>0$ and $\mathrm{d}=1$}

The model considered in section (2) with variations in the probabilities of inspection modes are treated. Any variation of the values of the probabilities $\mathrm{c}$ and $\mathrm{d}$ changes only some elements of $A_{0}, B_{1}, A_{1}$ and $Q^{\prime}$. In this model the CSP has the inspection probability in mode II is positive $\mathrm{c}>0$ and the inspection probability in mode III, $d=1$. This makes $\delta=\mathrm{p}$. The stationary probability vector $\mathrm{w}$ of the finite system generator matrix Q' is given by

$w_{k+r+1}=\left[\gamma^{r} p^{k} \mathrm{c} \mathrm{q}\right] /\left[\mathrm{c}\left(1-\mathrm{p} \gamma^{r}\right)+(1-\mathrm{c}) p^{k}\left(1-\gamma^{r}\right)\right]$;

$w_{i}=(1 / \mathrm{p})^{k-i}\left[\left(1 / \mathrm{p} \gamma^{r}\right)-1\right] w_{k+r+1}, \quad$ for $1 \leq \mathrm{i} \leq \mathrm{k}$ and $w_{i}=\left[1 / \gamma^{k+r-i+1}\right] w_{k+r+1}, \quad$ for $\mathrm{k}+1 \leq \mathrm{i} \leq \mathrm{k}+\mathrm{r}$.

(32)

The fraction of products that are inspected is then given by $\mathrm{f}=1-\sum_{i=1}^{r} w_{k+i}+\mathrm{c} \sum_{i=1}^{r} w_{k+i}$.

(33)

Whereas the defective products that go undetected as a fraction of all parts from (17) becomes (1-f) $\mathrm{q}=\mathrm{q}(1-$

c) $\sum_{i=1}^{r} w_{k+i}(34)$

This gives $\lambda\left[\mathrm{p}+\mathrm{q}(1-\mathrm{c}) \sum_{i=1}^{r} w_{k+i}\right]<\alpha \mu \mathrm{E}(\mathrm{N})$.

When the inequality (35) is satisfied the stationary distribution exists and the results presented in section (3) are all valid.

4.2. Model (B) Probabilities $\mathrm{c}=0$ and $\mathrm{d}>0$

In this model the CSP after the inspection mode I, changes to inspection mode II, with $\mathrm{c}=0$, where it is not inspecting the next $r$ products and for the $(\mathrm{r}+1)$-th product the CSP changes its inspection mode to III where the product is inspected with probability $\mathrm{d}>0$. The changes in the elements of the matrices $A_{0} B_{1}, A_{1}$ and $Q^{\prime}$ are as follows. In the matrices

$\lambda \mathrm{q} \mathrm{c}$ wherever it appears in them are to be replaced by 0 since $\mathrm{c}=0$ and $\lambda \gamma$ wherever it appears in them are to

be replaced by $\lambda$. The stationary probability vector $\mathrm{w}$ of the finite system generator matrix Q' is given by

$w_{i}^{\prime}=\left[\mathrm{d} \mathrm{q} / p^{k-i+1}\right] w^{\prime}{ }_{k+r+1}, \quad$ for $1 \leq \mathrm{i} \leq \mathrm{k}$;

$w_{k+i}^{\prime}=w_{k+r+1}^{\prime}$, for $1 \leq \mathrm{i} \leq \mathrm{r}$ and

$w^{\prime}{ }_{k+r+1}=p^{k} /\left[\mathrm{d}+p^{k}(1-\mathrm{d}+\mathrm{r})\right]$.

(36) The fraction of products that are inspected is then given by

$\mathrm{f}^{\prime}=1-\sum_{i=1}^{r+1} w_{k+i}^{\prime}+\mathrm{d} w_{k+r+1}^{\prime}$.

(37)

Whereas the defective products that go undetected as a

fraction of all parts is (1-f') $\mathrm{q}=\mathrm{q}\left[\sum_{i=1}^{r} w_{k+i}^{\prime}+(1-\right.$

d) $\left.w^{\prime}{ }_{k+r+1}\right]$. (38)

The stability condition for model (B) becomes

$\lambda\left[\mathrm{p}+\mathrm{q}(1-\mathrm{d}){w^{\prime}}_{k+r+1}+\mathrm{q} \sum_{i=1}^{r}{w^{\prime}}_{k+i}\right]<\alpha \mu \mathrm{E}(\mathrm{N})$.

(39)

When the inequality (39) is satisfied the stationary distribution exists and the results presented in section (3) are all valid. 


\section{NUMERICAL CASES}

Four numerical cases two each are treated for models (A) and (B) with different values for the CSP sizes $\mathrm{k}$ and $\mathrm{r}$ for inspection modes I and II and the results are presented in tables 1 and 3 for Model (A) and in tables 2 and 4 for Model (B). The values of the parameters are set as given below. For both the models, the production parameter $\lambda$ is 6 , the sale parameter $\mu$ is 5 , the normal sale allotment probability $\alpha$ is 0.8 , the production probability of good product $\mathrm{p}$ is 0.8 , the normal sale $\mathrm{N}$ has three sizes whose probabilities are respectively $p_{1}=0.6, p_{2}=0.3, p_{3}=0.1$. This gives the probability of a defective product $\mathrm{q}$ is 0.2 , expected sale size $\mathrm{E}(\mathrm{N})=1.5$ and the probability of catastrophic sale at a sale epoch $\beta$ is 0.2 . For Model (A) the probability of the CSP in inspection mode II, c is 0.5 and in inspection mode III, $\mathrm{d}$ is 1 . For Model (B) the probability of the CSP in inspection mode II, c is 0 and in inspection mode III, $\mathrm{d}$ is 0.5 . The results for the two models each for two cases with inspection sizes for modes I and II for $\mathrm{k}=2, \mathrm{r}=1$ and for $\mathrm{k}=3, \mathrm{r}=3$ are presented below. Table1 presents the results obtained for various measures for numerical case 1 of Model (A). The rate of entry $\lambda \mathrm{q}=1.2$ of defective products without any inspection policy reduces to 0.2232 due to the CSP. Defective products are not prevented from entering when the system state is $(\mathrm{i}, 2,1)$. There is a small difference in the probability values given in the last two rows corresponding to various states from the fourth decimal places since the iteration is stopped at E-05 level. E (Defectives) $=0.0647$. Table 2 gives the results for numerical case 2 for Model (B) when the inspection modes II and III have probability values $c=0$ and $d=0.5$. Defective products are not prevented from entering when the system state is (i, 2, 1) since $c=0$ in inspection mode II. It may be noted when the system is in state $(i, 3)$ only with probability $d$ a product is inspected. Here also all defectives are not prevented. There is some small difference in the probability values given in the last two rows corresponding to various states from the fourth decimal places since the iteration is stopped at E-05 level. In this example the rate of entry of defective products reduces from 1.2 to 0.263 due to the CSP. E (Defectives) = 0.9026.Table 3 exhibits results for numerical case for Model (A) for inspection size $\mathrm{k}=3$ for mode $\mathrm{I}$ and $\mathrm{r}=3$ as the size for inspection mode II. Here the rate of defective entry is reduced from 1.2 to 0.2996 . There is a small difference in the probabilistic values given in the last two rows in the corresponding to various states from the fourth decimal places since the iteration is stopped at E-05 level. E (Defectives) $=0.089$. The convergence is seen in the iteration 13 since difference norm is of order E-05. E(S) is nearly 2 and $\mathrm{E}$ (Defectives) is much less. Table 4 shows the results for Model (B) when the inspection mode II allows defectives and inspection mode III allows defectives with probability 1 -d. The iteration is done to get R (12) and norm is of order E- 05 . These four examples explain almost everything about the models studied. The merits of the CSP and its inspection modes II and III with the probabilities used for Model (A) and Model (B) can be seen from the fact that the values of $\mathrm{E}$ (Defectives) are more and the rate of entry of defectives are more for Model (B) as seen from the results presented. In table 5 it can be seen $\mathrm{E}(\mathrm{S}), \quad \mathrm{E}$ (Defectives), the defective entry rates and the standard deviation are less for Model (A) than Model (B). The coefficient of variation is not significantly different and almost same in the two models. But the defective entry rate and the expected defective stocks and the standard deviation are small for Model (A). Figure 1 presents all the risk measures concerned with the CSP mentioned in section (3) (viii) for comparisons of the results obtained for the four examples in a significant manner. The two models clearly reduce the rate of entry of defectives.

Table 1: Results for Model (A) for $k=2, r=1, c=0.5$ and $d=1$.

\begin{tabular}{|l|l|l|l|l|l|}
\hline $\mathrm{P}(.) \backslash$ states & $\pi(., 1,1)$ & $\pi(., 1,2)$ & $\pi(., 2,1)$ & $\pi(., 3)$ & $\begin{array}{l}\text { Defective } \\
\text { entry rate }\end{array}$ \\
\hline$\pi 0$ & 0.094585954 & 0.048109405 & 0.118731845 & 0.105003047 & 0.071239107 \\
\hline$\pi 1$ & 0.02525376 & 0.045345199 & 0.08561636 & 0.074903215 & 0.051369816 \\
\hline$\pi 2$ & 0.015508288 & 0.013569389 & 0.063739344 & 0.053358245 & 0.038243606 \\
\hline$\pi 3$ & 0.010099189 & 0.008406137 & 0.036663699 & 0.038179581 & 0.021998219 \\
\hline$\pi 4$ & 0.006326935 & 0.005442414 & 0.024951447 & 0.022514223 & 0.014970868 \\
\hline $\mathrm{P}($ States $>4)$ & 0.011141815 & 0.009439023 & 0.042306353 & 0.040805136 & 0.025383812 \\
\hline above sum & 0.16291594 & 0.130311566 & 0.372009048 & 0.334763446 & 0.223205429 \\
\hline w of Q' & 0.162790698 & 0.130232558 & 0.372093023 & 0.334883721 & 0.223255814 \\
\hline E(Stocks) & E(Defectives) $=$ & $(1-\mathrm{f}) \mathrm{q} \mathrm{E}(\mathrm{S})$ & \multicolumn{2}{|l|}{ Iteration for done to level 11 and stopped } \\
\hline 1.740183878 & 0.064773511 & & \multicolumn{2}{|l|}{ at difference norm = } & $6.01926 \mathrm{E}-05$ \\
\hline
\end{tabular}


Table 2: Results for Model $(B)$ for $k=2, r=1, c=0$ and $d=0.5$

\begin{tabular}{|c|c|c|c|c|c|}
\hline $\mathrm{P}(.) \backslash$ state & $(., 1,1)$ & $(., 1,2)$ & $(., 2,1)$ & $(., 3)$ & $\begin{array}{l}\text { Defective } \\
\text { entry rate }\end{array}$ \\
\hline$\pi 0$ & 0.039078735 & 0.020000091 & 0.135492235 & 0.13458365 & 0.243340872 \\
\hline$\pi 1$ & 0.010250582 & 0.018719885 & 0.095705767 & 0.094653623 & 0.171639094 \\
\hline$\pi 2$ & 0.006226699 & 0.005536092 & 0.068457896 & 0.066557915 & 0.122084224 \\
\hline$\pi 3$ & 0.004277545 & 0.003439428 & 0.044552613 & 0.046882486 & 0.081592627 \\
\hline$\pi 4$ & 0.002843367 & 0.002350923 & 0.030837744 & 0.030808384 & 0.055490323 \\
\hline $\mathrm{P}($ States $>4)$ & 0.005941192 & 0.00484116 & 0.063222647 & 0.064739341 & 0.114710781 \\
\hline sum of above & 0.06861812 & 0.05488758 & 0.438268901 & 0.438225398 & 0.78885792 \\
\hline w of Q' & 0.068493151 & 0.054794521 & 0.438356164 & 0.438356164 & 0.789041096 \\
\hline $\mathrm{E}($ Stocks $)=$ & 2.059275011 & $(1-f) q E(S)$ & \multicolumn{3}{|c|}{ Iteration done to level 10 and stopped } \\
\hline $\mathrm{E}($ defectives $)=$ & $(1-f) q E(S)$ & 0.902695895 & \multicolumn{2}{|c|}{ at difference norm $=$} & 5.04829E-05 \\
\hline
\end{tabular}

Table 3: Results for Model (A) for $k=3, r=3, c=0.5$ and $d=1$

\begin{tabular}{|c|c|c|c|c|c|c|c|c|c|}
\hline$P(.) \backslash$ state & $\pi \mathrm{i}$ & $\pi(i, 1,1)$ & $\pi(i, 1,2)$ & $\pi(i, 1,3)$ & $\pi(i, 2,1)$ & $\pi(i, 2,2)$ & $\pi(i, 2,3)$ & $\pi(i, 3)$ & Defect-entry rate \\
\hline$\pi 0=P(0$ product $)$ & 0.361471179 & 0.087246017 & 0.044352997 & 0.032829408 & 0.058058294 & 0.051614015 & 0.046097024 & 0.041273425 & 0.093461599 \\
\hline$\pi 1=P(1$ product $)$ & 0.229526221 & 0.023318404 & 0.041818705 & 0.024658517 & 0.041545308 & 0.036628088 & 0.032530938 & 0.029026261 & 0.066422601 \\
\hline$\pi 2=\mathrm{P}$ (2products) & 0.146256124 & 0.014376174 & 0.012506471 & 0.020264323 & 0.029625275 & 0.026018423 & 0.023011131 & 0.020454327 & 0.047192897 \\
\hline$\pi 3=P$ (3products) & 0.093570357 & 0.008920347 & 0.007745398 & 0.006717413 & 0.021330447 & 0.018258148 & 0.016200308 & 0.014398297 & 0.033473342 \\
\hline T4=P(4products) & 0.060296564 & 0.005778775 & 0.004845058 & 04179067 & 0.01 & 0.0 & 30 & 0.0 & 2199 \\
\hline$P(\#$ of products $>4)$ & 0.108879555 & 0.01048334 & 0.008819016 & 0.007413182 & 0.022143644 & 0.020670703 & 0.020219289 & 0.019 & 20182 \\
\hline sum of above & 1 & 0.150123057 & 0.120087645 & 0.096061909 & 0.184294205 & 0.165853014 & 0.149257481 & 0.13432269 & 0.29964282 \\
\hline sum of $w$ & 1 & 0.15004896 & 0.120039168 & 0.096031335 & 0.18432118 & 0.165889062 & 0.149300156 & 0.13437014 & 0.299706238 \\
\hline$E(S)$ & 1.784759428 & & \multicolumn{4}{|c|}{ Defective entry rate $=[\pi(., 2,2)+\pi(., 2,2)+\pi(., 2,3)](1-c) q \lambda$} & & & \\
\hline$E(D S)=E($ defective: & 0.089150589 & & Diff Norm= & 2.23173E-05 & & Iteration $13 \mathrm{fo}$ & & & \\
\hline
\end{tabular}

Table 4: Results for Model $(B)$ for $k=3, r=3, c=0$ and $d=0.5$

\begin{tabular}{|c|c|c|c|c|c|c|c|c|c|}
\hline P(i) \state & $\pi i$ & $\pi(\mid, 1,1)$ & $\pi(1,1,2)$ & $\pi(\mid, 1,3)$ & $\pi(1,2,1)$ & $\pi(1,2,2)$ & $\pi(1,2,3)$ & $\pi(\mid, 3)$ & Defect entry rate \\
\hline$\pi 0$ & 0.320054496 & 0.02533797 & 0.012890207 & 0.00955766 & 0.068337943 & $\begin{array}{ll}3 & 0.068109671\end{array}$ & 10.06796226 & $\begin{array}{ll}6 & 0.067858784\end{array}$ & 0.28600712 \\
\hline$\pi 1$ & 0.216487301 & 0.006632303 & $\begin{array}{l}3 \\
0.01212209\end{array}$ & 0.007156063 & 0.047958618 & $8 \quad 0.047692911$ & 10.047520264 & $4 \quad 0.047405052$ & .200249183 \\
\hline$\pi 2$ & 0.146910427 & 0.004055689 & 0.003559306 & 0.005865738 & 0.033634714 & $4 \quad 0.033409665$ & 0.033249844 & $\begin{array}{ll}4 & 0.033135471\end{array}$ & 0.140234349 \\
\hline$\pi 3$ & 0.100018613 & 0.002517394 & + 0.002199879 & 0.001917046 & 0.023652916 & $6 \quad 0.023334786$ & ; 0.023239668 & $\begin{array}{ll}8 & 0.023156924\end{array}$ & 0.098166999 \\
\hline$\pi 4$ & 0.068406114 & 0.001713773 & 0.001392134 & 0.001197949 & 0.015537136 & $6 \quad 0.016249826$ & 0.016169337 & $\begin{array}{l}7 \quad 0.016145959 \\
\end{array}$ & 0.067235134 \\
\hline$P($ States $>4)$ & 0.148123048 & 0.003715253 & $\begin{array}{l}3 \\
0.00300463\end{array}$ & 0.002432911 & 0.034133098 & $\begin{array}{ll}8 & 0.034409813 \\
\end{array}$ & 0.035017772 & $2 \quad 0.03540957$ & 0.145518562 \\
\hline sumof above & 1 & 0.043972383 & 0.035168248 & 0.028127367 & 0.223254425 & $5 \quad 0.223206672$ & 0.223159146 & 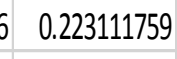 & 0.937411347 \\
\hline w of $Q^{\prime}$ & 1 & 0.043630017 & 0.034904014 & 0.027923211 & 0.223385689 & $\begin{array}{ll}9 & 0.223385689 \\
\end{array}$ & 0.223385689 & $\begin{array}{ll}9 & 0.223385689\end{array}$ & 0.93821989 \\
\hline$E(S)$ & 2.145330722 & \multicolumn{6}{|c|}{ Defectives entry rates $=\lambda q[\pi(., 2,1)+\pi(., 2,2)+\pi((., 2,3)]+\lambda q d \pi(., 3)$} & & \\
\hline \multicolumn{2}{|c|}{$E($ Defectives):(1-f)qE(S)= } & 0.10226331 & Diff norm= & \multicolumn{3}{|c|}{ 5.50019E-05 Iteration 12 for $R$} & & & \\
\hline
\end{tabular}


Table 5: Results of Four Cases Listed for Comparison

\begin{tabular}{|l|l|l|l|l|}
\hline & Case1 & Case2 & Case3 & Case4 \\
\hline$E(S)$ & 1.740183878 & 2.059275011 & 1.784759428 & 2.145330722 \\
\hline E(DS) & 0.064773511 & 0.902695895 & 0.089150589 & 0.10226331 \\
\hline $\begin{array}{l}\text { DE } \\
\text { Rate }\end{array}$ & 0.223255814 & 0.789041096 & 0.299706238 & 0.938219895 \\
\hline VAR (S) & 4.795628672 & 6.357062209 & 5.027621141 & 6.816643507 \\
\hline Std.dev & 2.189892388 & 2.52132152 & 2.242235746 & 2.610830341 \\
\hline CV & 1.258425857 & 1.224373387 & 1.2563238 & 1.216982684 \\
\hline$\pi 0$ & 0.366430251 & 0.329154712 & 0.361471179 & 0.320054496 \\
\hline
\end{tabular}

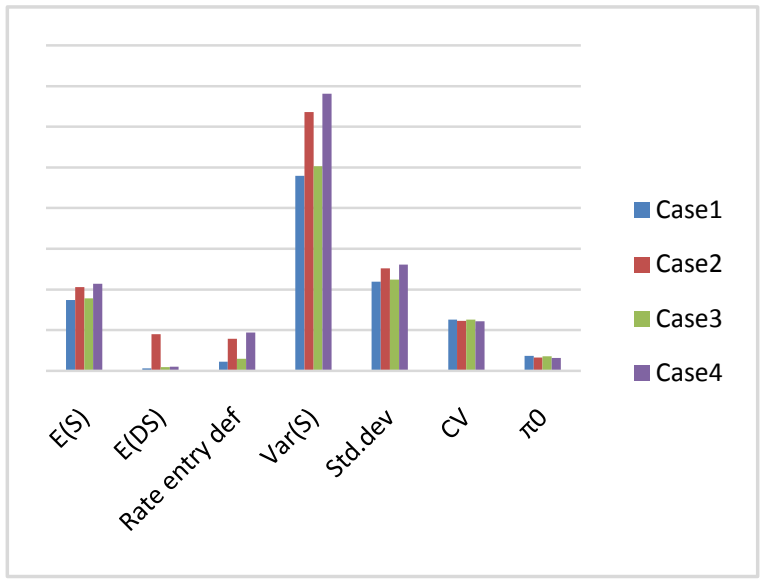

Figure 1: Performance Measures for Four Cases

\section{CONCLUSION}

In this paper inspection by a continuous sampling plan (CSP) is presented to prevent the entry of defective products into stocks for sale. The CSP considered here has three modes. In mode I every single product is inspected until $\mathrm{k}$ consecutive good products are found. At this point the CSP changes its inspection mode to II of inspecting the next $r$ products where every product is inspected with probability $\mathrm{c}$ until a defective product appears. When a defective one appears, the CSP changes its mode to I and if no defective product is noticed in those $\mathrm{r}$ products, the CSP changes to mode III where the product is inspected with probability $d$. If it is rejected, the CSP changes to mode I and if it is not rejected then it changes to mode II. In the above continuous sampling plan, the probabilistic inspection procedures reduce the number of inspections considerably with a view to reduce the inspection cost. Giving different values for $\mathrm{c}$ and $\mathrm{d}$ two different models are discussed. Both models reduce the rate of entry of defective items. All the risks measures involved in the CSP are studied in the numerical cases. Model (A) seems to be suitable due to statistical consideration if cost is not playing a role. Risks and associated cost with it are at times over looked for perfection.

\section{ACKNOWLEDGMENT}

The author thanks Dr. R. Ramanarayanan and ANSYS Inc., USA, for providing guidance and facilities. The contents of the article published are the responsibilities of the author

\section{REFERENCES}

[1] H.F.Dodge (1943). A sampling inspection plan for continuous production. Annals of Statistics, 14, 264-279

[2] G.K. Mytalas and M.A. Zazanis, (2007), Delay analysis of continuous sampling plans www.aueb.gr/pympe/hercma/ proceedings2007/H07FULL-PAPERS

[3] Albert H. Bowker, (1956) Proceedings of the Third Berkeley Symposium projecteuclid.org/download/pdf1/euclid.bsmsp/1200511857

[4] Ivo Adan and Jacques Resing,(2001) Queueing theory,http//wwwhome.math.utwente.nl/scheinhardwrw/ queueingdictaat.pdf

[5] Kenblock, (2012) Business statistics for contemporary decision making, John Wiley \& sons Inc.

[6] James c. Cox and Vjollca Sadiraj, (2009) On the coefficient of variation as a measure of risk of sensitivity, http://excen.gsu.edu/ working papers /GSU_EXCEN_WP_2009-06.pdf

[7] Rockefeller.R.T, (2007) Coherent approaches to risk in optimization under uncertainty, Tutorials in Operations Research INFORMS, 38-61, http://www.math.washington.edu/-rt/papers.html.

[8] Neuts. M.F. (1981).Matrix-Geometric Solutions in Stochastic Models: An algorithmic Approach, The Johns Hopkins Press, Baltimore

[9] Bini, Latouche and Mein. Bini, (2005). Numerical methods for structured Markov chains, Oxford Univ. Press, Oxford

[10] Latouche and Ramaswami (1998). Introduction to Matrix Analytic Methods in Stochastic Modeling, SIAM. Philadelphia 